\title{
Heun Method Using to Solve System of NonLinear Functional Differential Equations
}

\author{
Shymaa Hussain Salih*
}

Date of acceptance 22/8/2007

\begin{abstract}
:
In this paper Heun method has been used to find numerical solution for first order nonlinear functional differential equation. Moreover, this method has been modified in order to treat system of nonlinear functional differential equations .two numerical examples are given for conciliated the results of this method.
\end{abstract}

Keywords: Nonlinear Functional Differential Equation; Numerical Method; Heun Methods.

\section{Introduction:}

The functional differential equation "FDE" is an equation in an unknown function $\mathrm{y}(\mathrm{t})$ and some of its derivatives are evaluated at arguments that differ in any of fixed number of values $\tau_{1}, \tau_{2}, \ldots, \tau_{n}$

The general form of nonlinear FDE is given by

$y_{i}^{\prime}=f_{i}\left(t, y_{1}(t), \ldots, y_{n}(t), y_{1}\left(t-\tau_{1}\right), \ldots, y_{n}\left(t-\tau_{n}\right), \ldots\right)$

$i=1,2, \ldots, n$

where $\tau_{1}, \tau_{2}, \ldots, \tau_{n}$ are positive real numbers,

$f_{i} \in C\left[R^{+} \times R^{n}, R\right] \quad i=1,2, \ldots, n$ with

exception triviality solution by condition $\sup \left\{\sum\left|y_{i}\right|\right\}>0$.

The general linear first order functional differential equation with can be presented in the form [1]

$a_{0} y^{\prime}(t)+a_{1} y^{\prime}(t-\tau)+b_{0} y(t)+b_{1} y(t-\tau)=g(t)$

where $\mathrm{g}(\mathrm{t})$ is a given continuous function and $\tau$ is a time delay and $a_{0}, a_{1}, b_{0}, b_{1}$ are constants.
We can distinguish between three types of functional differential equations, which are:

1. Retarded functional differential equation [2], which is obtained when:

$\left(b_{1} \neq 0, a_{1}=0\right)$ in eq.(1) the delay comes in y only and (1) takes the form $a_{0} y^{\prime}(t)+b_{0} y(t)+b_{1} y(t-\tau)=g(t)$

2. Neutral functional differential equation [3], which is obtained when:

$$
\left(a_{1} \neq 0, b_{1}=0\right) \text { in eq.(1) the }
$$

delay comes in $y^{\prime}$ only and (1) takes the form

$a_{0} y^{\prime}(t)+a_{1} y^{\prime}(t-\tau)+b_{0} y(t)=g(t)$

3. Mixed functional differential equation [4], (sometimes called advanced type), which is obtained where $\left(a_{1} \neq 0, b_{1} \neq 0\right)$, which means a combination of the previous two types.

\section{Heun Method: -}

Heun method is one of the simplest numerical methods. Peitgen and Glashoff [5] used this method to find the numerical solution of nonlinear differential equations, while Vladimir and Milan [6] applied this method to treat nonlinear ordinary differential equations. 
Consider the following first order nonlinear differential equation

$y^{\prime}=f(t, y)$ with $\quad y\left(t_{0}\right)=y_{0}$

Heun method for eq. (2) is:

$y_{n+1}=y_{n}+\frac{h}{4}\left(H_{1}+3 H_{3}\right)$

Where

$$
\begin{aligned}
& H_{1}=f\left(t_{n}, y\left(t_{n}\right)\right) \\
& H_{2}=f\left(t_{n}+h / 3, y\left(t_{n}\right)+h / 3 H_{1}\right) \\
& H_{3}=f\left(t_{n}+2 / 3 h, y\left(t_{n}\right)+2 / 3 h H_{2}\right)
\end{aligned}
$$

In this work the idea of Heun method has been used to treat both nonlinear functional differential equations as well as system of nonlinear functional differential equations numerically .

\begin{tabular}{l}
1 Solution of $a 1^{\text {st }}$ Order \\
\hline Nonlinear
\end{tabular} Nonlinear

\section{Differential Equation}

\section{Using Heun Method}

Consider the following first order nonlinear functional differential equation

$y^{\prime}(t)=f\left(t, y(t), y(t-\tau), y^{\prime}(t-\tau)\right) ; \quad t \in\left[t_{0}, \infty\right)$

with initial function

$y(t)=\phi(t) \quad$ for $\quad t_{0}-\tau \leq t \leq t_{0}$

eq.(5) may be solved by Heun method if we use the initial function

$y^{\prime}(t)=f\left(t, y(t), \phi(t-\tau), \phi^{\prime}(t-\tau)\right)$

with initial condition

$$
y\left(t_{0}\right)=\phi\left(t_{0}\right)
$$

When applying Heun method equation (6) using eqs. (3) and (4) we get the following formula.

$$
y_{j+1}=y_{j}+\frac{h}{4}\left(H_{1}+3 H_{3}\right)
$$

where
$H_{1}=f\left(t_{j}, \phi\left(t_{j}\right), \phi\left(t_{j}-\tau\right), \phi^{\prime}\left(t_{j}-\tau\right)\right)$

$H_{2}=f\left(t_{j}+h / 3, \phi\left(t_{j}\right)+h / 3 H_{1}, \phi\left(t_{j}-\tau\right)+h / 3 H_{1}, \phi^{\prime}\left(t_{j}-\tau\right)+h / 3 H_{1}\right)$

$H_{3}=f\left(t_{j}+2 / 3 h, \phi\left(t_{j}\right)+2 / 3 h H_{2}, \phi\left(t_{j}-\tau\right)+2 / 3 h H_{2}, \phi^{\prime}\left(t_{j}-\tau\right)+2 / 3 h H_{2}\right)$ for $\mathrm{j}=0,1,2, \ldots, \mathrm{n}$ where $\mathrm{n}$ is the number of steps

\section{Algorithm (AHe1)}

The numerical solution of $1^{\text {st }}$ order nonlinear functional differential equation using Heun method is computed as follows: -

Step1: set $\mathrm{h}=(\mathrm{b}-\mathrm{a}) / \mathrm{n}$

Step2: set $t_{j}=a+j h$ for each $j=0,1, \ldots, n$

Step3: compute

$$
H_{1}=f\left(t_{j}, \phi\left(t_{j}\right), \phi\left(t_{j}-\tau\right), \phi^{\prime}\left(t_{j}-\tau\right)\right)
$$

Step4: compute

$H_{2}=f\left(t_{j}+h / 3, \phi\left(t_{j}\right)+h / 3 H_{1}, \phi\left(t_{j}-\tau\right)+h / 3 H_{1}, \phi^{\prime}\left(t_{j}-\tau\right)+h / 3 H_{1}\right)$

Step5:compute

$H_{3}=f\left(t_{j}+2 / 3 h, \phi\left(t_{j}\right)+2 / 3 h H_{2}, \phi\left(t_{j}-\tau\right)+2 / 3 h H_{2}, \phi^{\prime}\left(t_{j}-\tau\right)+2 / 3 h H_{2}\right)$

Step6: compute

$y_{j+1}=y_{j}+\frac{h}{4}\left(H_{1}+3 H_{3}\right)$

\section{Solution of a System of $1^{\text {st }}$ Order NonLinear Functional Differential Equations \\ Using Heun Method}

Consider a system of first order nonlinear functional differential equation Such as:

$y_{i}^{\prime}=f_{i}\left(t, y_{1}(t), \ldots, y_{n}(t), y_{1}\left(t-\tau_{1}\right), \ldots, y_{n}\left(t-\tau_{n}\right), y_{1}^{\prime}\left(t-\tau_{1}\right), \ldots, y_{n}^{\prime}\left(t-\tau_{n}\right)\right), \quad t \in\left[t_{0}, \infty\right)$

$i=1,2, \ldots, n$

with initial functions

$$
\begin{array}{ccc}
y_{1}(t)=\phi_{1}(t) & \text { for } & t_{0}-\tau_{1} \leq t \leq t_{0} \\
\vdots & & \vdots \\
y_{n}(t)=\phi_{n}(t) & \text { for } & t_{0}-\tau_{n} \leq t \leq t_{0}
\end{array}
$$


Equation (7) may be solved by Heun method if

$y^{\prime}(t)=f\left(t, y_{1}(t), \ldots, y_{n}(t), \phi_{1}\left(t-\tau_{1}\right), \ldots, \phi_{n}\left(t-\tau_{n}\right), \phi_{1}^{\prime}\left(t-\tau_{1}\right), \ldots, \phi_{n}^{\prime}\left(t-\tau_{n}\right)\right)$

with initial condition

$y_{1}\left(t_{0}\right)=\phi_{1}\left(t_{0}\right), \ldots ., y_{n}\left(t_{0}\right)=\phi_{n}\left(t_{0}\right)$

For treating system of nonlinear functional differential equation eq. (3) and (4) we have

$y_{i j+1}=y_{i j}+\frac{h}{4}\left(H_{1 i}+3 H_{3 i}\right)$

where

$H_{1 i}=f_{i}\left(t_{j}, \phi_{1}\left(t_{j}\right), \ldots, \phi_{m}\left(t_{j}\right), \phi_{1}\left(t_{j}-\tau_{1}\right), \ldots, \phi_{m}\left(t_{j}-\tau_{m}\right), \phi_{1}^{\prime}\left(t_{j}-\tau_{1}\right), \ldots, \phi_{m}^{\prime}\left(t_{j}-\tau_{m}\right)\right)$

$H_{2 i}=f_{i}\left(t_{j}+h / 3, \phi_{1}\left(t_{j}\right)+h / 3 H_{11}, \ldots, \phi_{m}\left(t_{j}\right)+h / 3 H_{1 m}, \phi_{1}\left(t_{j}-\tau_{1}\right)+h / 3 H_{11}, \ldots\right.$ $\left.\phi_{m}\left(t_{j}-\tau_{m}\right)+h / 3 H_{1 m}, \phi^{\prime}\left(t_{j}-\tau_{1}\right)+h / 3 H_{11}, \ldots, \phi^{\prime}\left(t_{j}-\tau_{m}\right)+h / 3 H_{1 m}\right)$

$H_{3 i}=f_{i}\left(t_{j}+2 / 3 h, \phi_{1}\left(t_{j}\right)+2 / 3 h H_{21}, \ldots, \phi_{m}\left(t_{j}\right)+2 / 3 h H_{2 m}, \phi_{1}\left(t_{j}-\tau_{1}\right)+2 / 3 h H_{21}, \ldots\right.$, $\left.\phi_{m}\left(t_{j}-\tau_{m}\right)+2 / 3 h H_{2 m}, \phi_{1}^{\prime}\left(t_{j}-\tau_{1}\right)+2 / 3 h H_{21}, \ldots, \phi_{m}^{\prime}\left(t_{j}-\tau_{m}\right)+2 / 3 h H_{2 m}\right)$

for $\mathrm{j}=0,1, \ldots, \mathrm{n}, \mathrm{i}=0,1, \ldots, \mathrm{m}$

\section{Algorithm (AHes2)}

The numerical solution of System $1^{\text {st }}$ order nonlinear functional differential equation using Heun method is computed as follows: -

Step1: set $h=(b-a) / n$

Step 2:Set $t_{j}=a+j h$ for each $j=0,1, \ldots, n$

Step 3: Compute

$H_{1 i}=f_{i}\left(t_{j}, \phi_{1}\left(t_{j}\right), \ldots, \phi_{m}\left(t_{j}\right), \phi_{1}\left(t_{j}-\tau_{1}\right), \ldots, \phi_{m}\left(t_{j}-\tau_{m}\right), \phi_{1}^{\prime}\left(t_{j}-\tau_{1}\right), \ldots, \phi_{m}^{\prime}\left(t_{j}-\tau_{m}\right)\right)$

Step 4: Compute

$H_{2 i}=f_{i}\left(t_{j}+h / 3, \phi_{1}\left(t_{j}\right)+h / 3 H_{11}, \ldots, \phi_{m}\left(t_{j}\right)+h / 3 H_{1 m}, \phi_{1}\left(t_{j}-\tau_{1}\right)+h / 3 H_{11}, \ldots\right.$, $\left.\phi_{m}\left(t_{j}-\tau_{m}\right)+h / 3 H_{1 m}, \phi^{\prime}\left(t_{j}-\tau_{1}\right)+h / 3 H_{11}, \ldots, \phi^{\prime}\left(t_{j}-\tau_{m}\right)+h / 3 H_{1 m}\right)$

Step5: compute

$H_{3 i}=f_{i}\left(t_{j}+2 / 3 h, \phi_{1}\left(t_{j}\right)+2 / 3 h H_{21}, \ldots, \phi_{m}\left(t_{j}\right)+2 / 3 h H_{2 m}, \phi_{1}\left(t_{j}-\tau_{1}\right)+2 / 3 h H_{21}, \ldots\right.$, $\left.\phi_{m}\left(t_{j}-\tau_{m}\right)+2 / 3 h H_{2 m}, \phi_{1}^{\prime}\left(t_{j}-\tau_{1}\right)+2 / 3 h H_{21}, \ldots, \phi_{m}^{\prime}\left(t_{j}-\tau_{m}\right)+2 / 3 h H_{2 m}\right)$

Step6: compute

$$
y_{i j+1}=y_{i j}+\frac{h}{4}\left(H_{1 i}+3 H_{3 i}\right)
$$

\section{Numerical Examples: Example (1):}

Consider the $1^{\text {st }}$ order nonlinear functional differential equation: -

with initial function $y^{\prime}(t)=\frac{8}{t+1} y\left(t-\left(\frac{t}{2}+\frac{1}{2}\right)\right), \quad t \geq 0$

$y(t)=(t+1)^{2} \quad-1 \leq t \leq 0$

While the exact solution is

$y(t)=(t+1)^{2} \quad 0 \leq t \leq 1$

Take $\mathrm{n}=10, \mathrm{~h}=0.1$ and $t_{i}=i h, \mathrm{i}=0,1 \ldots \mathrm{n}$ the result is obtained by the running program $\mathrm{AHe} 1$.

Table (1) presents comparison between the exact and numerical solution which depend on least square error.

\begin{tabular}{|c|c|c|}
\hline \multicolumn{3}{|c|}{ Heun Method } \\
\hline $\mathrm{t}$ & Exact & $\mathrm{AHe} 1$ \\
\hline 0 & 1 & 1 \\
\hline 0.1 & 1.2100 & 1.2100 \\
\hline 0.2 & 1.4400 & 1.4400 \\
\hline 0.3 & 1.6900 & 1.6900 \\
\hline 0.4 & 1.9600 & 1.9600 \\
\hline 0.5 & 2.2500 & 2.2500 \\
\hline 0.6 & 2.5600 & 2.5600 \\
\hline 0.7 & 2.8900 & 2.8900 \\
\hline 0.8 & 3.2400 & 3.2400 \\
\hline 0.9 & 3.6100 & 3.6100 \\
\hline 1 & 4.000 & 4.000 \\
\hline \multicolumn{2}{|c|}{ L.S.E. } & $1.528 \mathrm{e}-30$ \\
\hline
\end{tabular}

Table (1) Solution of Example (1)

\section{Example (2): -}

Consider the following system of two nonlinear functional differential equations: -

$$
\begin{aligned}
& y_{1}^{\prime}(t)=1-y_{1}\left(\operatorname{Exp}\left(1-\frac{1}{t}\right)\right), \quad t>0 \\
& y_{2}^{\prime}(t)=-y_{2}\left(1-\frac{1}{t}\right) \quad y_{2}^{2}(t) \quad y_{1}\left(\operatorname{Exp}\left(1-y_{2}(t)\right)\right), \quad t>0
\end{aligned}
$$

with initial functions

$y_{1}(t)=\ln t$

$y_{2}(t)=\frac{1}{t} \quad 1 \leq t \leq 2$

the analytical solution

$y_{1}(t)=\ln t \quad 0<t \leq 1$

$y_{2}(t)=\frac{1}{t}$

Take $\mathrm{n}=10, \mathrm{~h}=0.1$ and $t_{i}=i h, \mathrm{i}=0,1 \ldots \mathrm{n}$ the result are obtained by running the program AHes2. 
Table (2) presents a comparison between the exact and the numerical solution which depends on least square error.

\begin{tabular}{|c|c|c|c|c|}
\hline \multicolumn{5}{|c|}{ Heun Method } \\
\hline $\mathrm{t}$ & Exact1 & AHes1 & Exact2 & AHes2 \\
\hline 1 & 0.000 & 0.000 & 1.000 & 1.000 \\
\hline 1.1 & 0.0953 & 0.0953 & 0.9091 & 0.9091 \\
\hline 1.2 & 0.1823 & 0.1823 & 0.8333 & 0.8333 \\
\hline 1.3 & 0.2624 & 0.2624 & 0.7692 & 0.7692 \\
\hline 1.4 & 0.3365 & 0.3365 & 0.7143 & 0.7143 \\
\hline 1.5 & 0.4055 & 0.4055 & 0.6666 & 0.6666 \\
\hline 1.6 & 0.4700 & 0.4700 & 0.6250 & 0.6250 \\
\hline 1.7 & 0.5306 & 0.5306 & 0.5882 & 0.5882 \\
\hline 1.8 & 0.5878 & 0.5878 & 0.5555 & 0.5555 \\
\hline 1.9 & 0.6419 & 0.6419 & 0.5263 & 0.5263 \\
\hline 2 & 0.6932 & 0.6932 & 0.5000 & 0.5000 \\
\hline \multicolumn{2}{|c|}{ L.S.E } & $4.1942 \mathrm{e}-10$ & L.S.E & $4.7235 e-9$ \\
\hline
\end{tabular}

Table (2) Solution of Example (2)

\section{Conclusion:}

A Heun method has been presented for solving a system of non-linear functional differential equations. The results show remarkable improvement in the least square errors from which we conclude that:

1. The good approximation depends on the size of $h$, if $h$ is decreased then the number of division points increase and L.S.E approaches zero.
2. Heun method provides convenient and efficient way for solving first order non-linear functional differential equations as well as system of non-linear functional differential equations numerically and finding accurate results.

\section{References:}

1. Byung, Kookim, 1972" Piecewise linear Dynamic systems with time Delay",printed in California.

2. Cryer,W.,1972 "Numerical method for functional differential equations", Academic press ,N.Y.

3. Gyori,Hartung ,Turi J., "On Numerical Solution for a class of nonlinear functional differential equations with time -and statedependent delays", www.ma.man.ac.uk/nare parts .

4. Hussain ,Mohamad A.,2004 "First order Nonlinear Neutral Delay Differential Equations", J. of UmSalama for sciences.

5. Peitgen, Glash H., 1981"Numerical Solution of Nonlinear Equations",Berlin Heidelberg,Germany. Vladimir,Milan K.,1983 "Numerical Solution of Nonlinear Boundary Value Problems with Applications" ,PrinticeHall Inc,EngleWood Cliffs.

\section{أستخدام طريقة هون لحل منظومة معادلات الدوال التفاضلية غير الخطية}

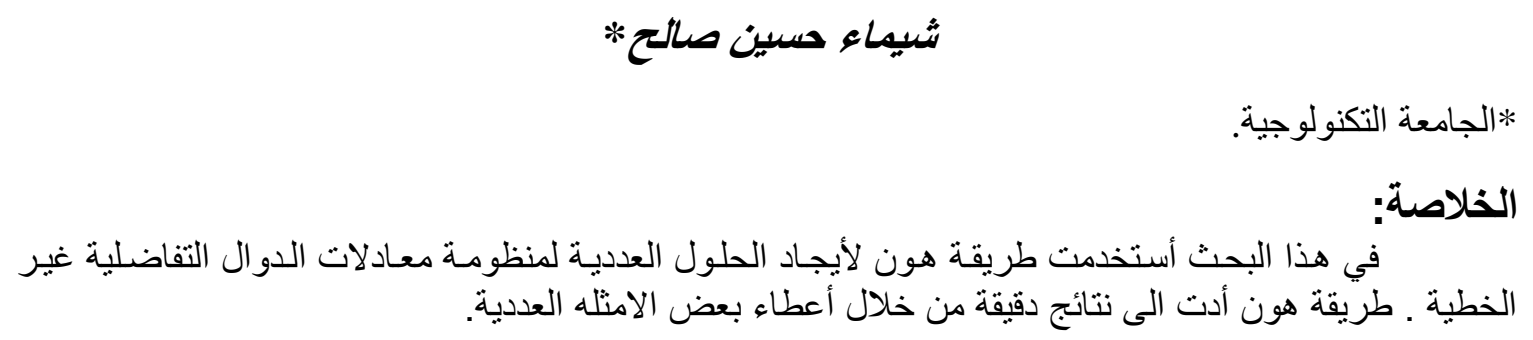

